\title{
Analysis of Blood Pressure after and of Exercise Training Competitor
}

\section{Rubem Machado Filho ${ }^{1^{*}}$, Leonardo Amado Sohr Cardoso ${ }^{2}$ and Fábio de Albuquerque Gonçalves ${ }^{2}$}

${ }^{1}$ Doctor of Human Movement Sciences (UNIMEP), Salgado de Oliveira University, Brazil

${ }^{2}$ Physical Education Specialist by the Gama Filho University, Brazil

*Corresponding author: Rubem Machado Filho, Doctor of Human Movement Sciences (UNIMEP), Salgado de Oliveira University, UNIVERSO, São Gonçalo, Rio de Janeiro, Brazil

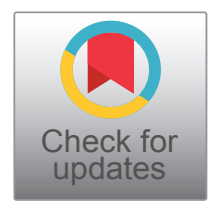

\begin{abstract}
Are called concurrent training programs that combine strength training and aerobic endurance in the same training session. This study aimed to verify the behavior of blood pressure during and after a training session competitor. We studied 10 subjects aged $30.8 \pm 12.8$ (19 to 53 years) (4 hypertensive and normotensive 6 ) of both genders ( 5 men and 5 women). The statistical analysis was performed using software (Primer for Windows, version 4.0.0.0, McGraw-Hill, USA) using paired t-test, giving the significance level of $p<$ 0.05 for SBP and DBP. The results obtained in this study indicate HPE for 60 minutes when performed concurrent training in question and suggests a reduction in BP similar to aerobic training, but with a slightly greater reduction in DBP.
\end{abstract}

\section{Keywords}

Cardiovascular physiology, Blood pressure, ConCurrent training

\section{Introduction}

Concurrent training is the training programs that combine strength and aerobic resistance in the same training session [1]. Strength and endurance training can be carried out concurrently to improve performance in sports, as well as for rehabilitation of injuries and cardiovascular diseases [2].

Physical activity programs have been shown to decrease SBP and DBP, both for hypertensive and normotensive individuals [3]. A few years ago, more specifically until the 1990 s, the recommendation of exercise for people with some chronic disease, mainly of a cardiovascular nature, was restricted to aerobic training. However, conducting studies involving counterresistance exercise identified chronic adaptations that aerobic training did not cease yesterday [4]. Regular physical exercise causes important autonomic and hemodynamic adaptations that will influence the cardiovascular system, with the aim of maintaining cellular homeostasis in the face of increased metabolic demands [5]. The mechanisms that guide pressure drop after physical training are related to hemodynamic, humoral and neural factors [5].

Several studies have shown that physical activities with aerobic characteristics show a significant reduction in blood pressure levels after exercise, whereas after resistance exercises, controversial results have been shown, such as elevation, maintenance, or even reduction in blood pressure after exercise [6]. It seems clear that the magnitude and duration of the pressure drop can be influenced by several factors, such as the sample studied (normotensive or hypertensive), the type, intensity and duration of the exercise [7].

The physiological mechanisms that can mediate the adaptive responses of concurrent training remain speculative, but they seem to indicate changes in neural recruitment patterns, attenuation in muscle hypertrophy or both [8]. Concurrent training practice during an exercise session, in view of the hemodynamic characteristics and responses that each activity has, may result in a different blood pressure behavior from those already known.

The present study aimed to verify the behavior of 
blood pressure during and after a concurrent training session.

\section{Methodology}

Ten individuals aged $30.8 \pm 12.8$ (19 to 53-years-old) ( 4 hypertensive and 6 normotensive) of both genders ( 5 men and 5 women) were studied. The individuals studied were physically active and practiced aerobic exercise and resistance at least three times a week for at least six months. Osteomyoarticular complications that limited or prevented the exercise were excluded from the experiment. Only hypertensive volunteers who were under the influence of pharmacological drugs according to medical guidelines were accepted. All individuals voluntarily participated in the experiment and signed a consent form.

Blood pressure was measured using a pressure device (aneroid sphygmomanometer, Premium, China) with a high-precision manometer with Japanese technology and a stethoscope (simple, Premium, China). The exercises were performed on a treadmill (9100HR, Life Fitness, USA), leg press washer (MG-541, Buick, Brazil), pulley 4 stations (MG-557, Buick, Brazil), bench bench (BA-113, Buick, Brazil) and dumbbells.

Blood Pressure (PB) was checked using the auscultatory method. This method uses a stethoscope and a device called a sphygmomanometer, composed of an inflatable arm cuff connected to a column of mercury or an aneroid marker (pointer). The measurement occurs through arterial occlusion due to cuff inflation, correlating the auscultation of the heartbeat with the value recorded in the mercury column or by the hand. The sounds heard during the measurement procedure are called Korotkoff noises, being classified into five phases [9]. The intensity of the exercises was measured by Borg's subjective effort scale, as it is easy to apply and the safety of the method for the hypertensive population that participated in the study.

Prior to the beginning of the study, its objective and the procedure adopted to determine the intensity of the exercises as well as the correct way to perform them were explained. The participants' resting Blood Pressure (BP) was measured and then aerobic exercise started on the treadmill for 30 minutes at an intensity considered moderate by them according to the Borg scale. At the end of the aerobic exercise, the participants were allowed to rest for 5 minutes, at the end of that interval the BP was measured and then the resistance training started.

Counter-resistance exercises consisted of bench press with dumbbells on a straight bench, pulled forward from the pulley with hands in pronation and inclined leg press. The dumbbell supine exercise was performed with the individual lying on the horizontal bench, feet flat against the floor, elbows flexed at $90^{\circ}$ aligned with the shoulders and hands in pronation; develop until the dumbbells are face to face; lower the dumbbells to the chest level by flexing your elbows. The pull from the front was performed sitting in front of the device, lower limbs supported, a secure bar in pronation and with the hands more than shoulder width apart; pull the bar to the suprasternal notch projecting the elbows down and return to the starting position. The leg press was performed with the feet positioned in the middle of the platform with a medium spacing; unlock the safety lock and flex the knee and hip; return to the starting position. For each exercise, 3 sets of 10 repetitions were performed with a 1-minute interval between sets and a 3-minute interval between exercises. BP was measured 3 minutes after the end of the third set of each exercise and 1 hour after the end of the session.

Statistical analysis of the data was performed using software (Primer for Windows version 4.0.0.0, McGrawHill, USA) using the paired t-test, obtaining a significance level of $p<0.05$ for PAS and PAD.

\section{Results}

HPE of SBP $(p<0.05)$ and DBP $(p<0.05)$ was observed in the concurrent training session in all recovery intervals when compared to pre-exercise rest values. The initial (118.2) and final (107.2) mean SBP showed standard deviation 11.09 and 13.44 respectively, while the average (73) and final (66.4) mean DBP presented the values 12, 01 and 9.65 standard deviation. The graphs in Figure 1 and Figure 2 show the behavior of the mean SBP and DBP in the post-exercise recovery intervals.

\section{Discussion}

This study verified the behavior of Blood Pressure (BP) during and after a concurrent training session composed of aerobic exercise and counter-resistance. Post-Exercise Hypotension (PEH) was observed in all individuals, when the resting values were compared with the values after each exercise and 60 minutes after the end of the training session. There was a drop of up to $18 \mathrm{mmHg}$ in Systolic Blood Pressure (SBP) and 14 $\mathrm{mmHg}$ in Diastolic Blood Pressure (DBP), however, DBP showed a decreasing pattern of $s$ values while SBP had oscillations throughout the training session.

The various studies that address the relationship between BP and physical exercise have focused mainly on dynamic aerobic exercise, that is, exercises of a continuous nature that require a prolonged period of time and involve large muscle groups in their execution [7]. The counter-resistance exercise, or resistance, consists of a local muscular work, which uses overloads such as weight of machines, bars, washers. It is performed with moderate loads and frequent repetitions, presenting pauses between executions, and, therefore, characterized as discontinuous effort. 


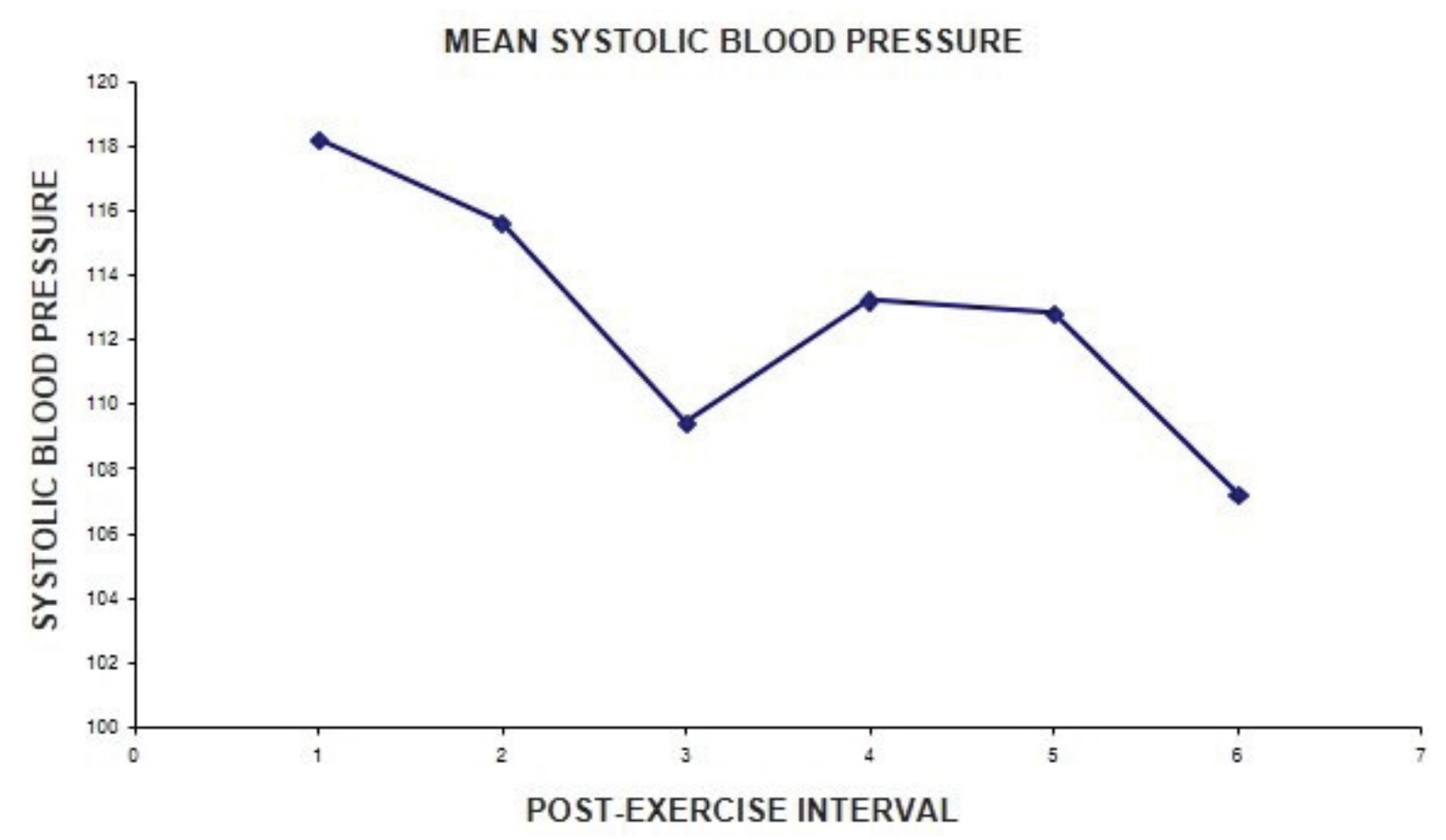

Figure 1: Average variation of systolic blood pressure in individuals during a concurrent training session.

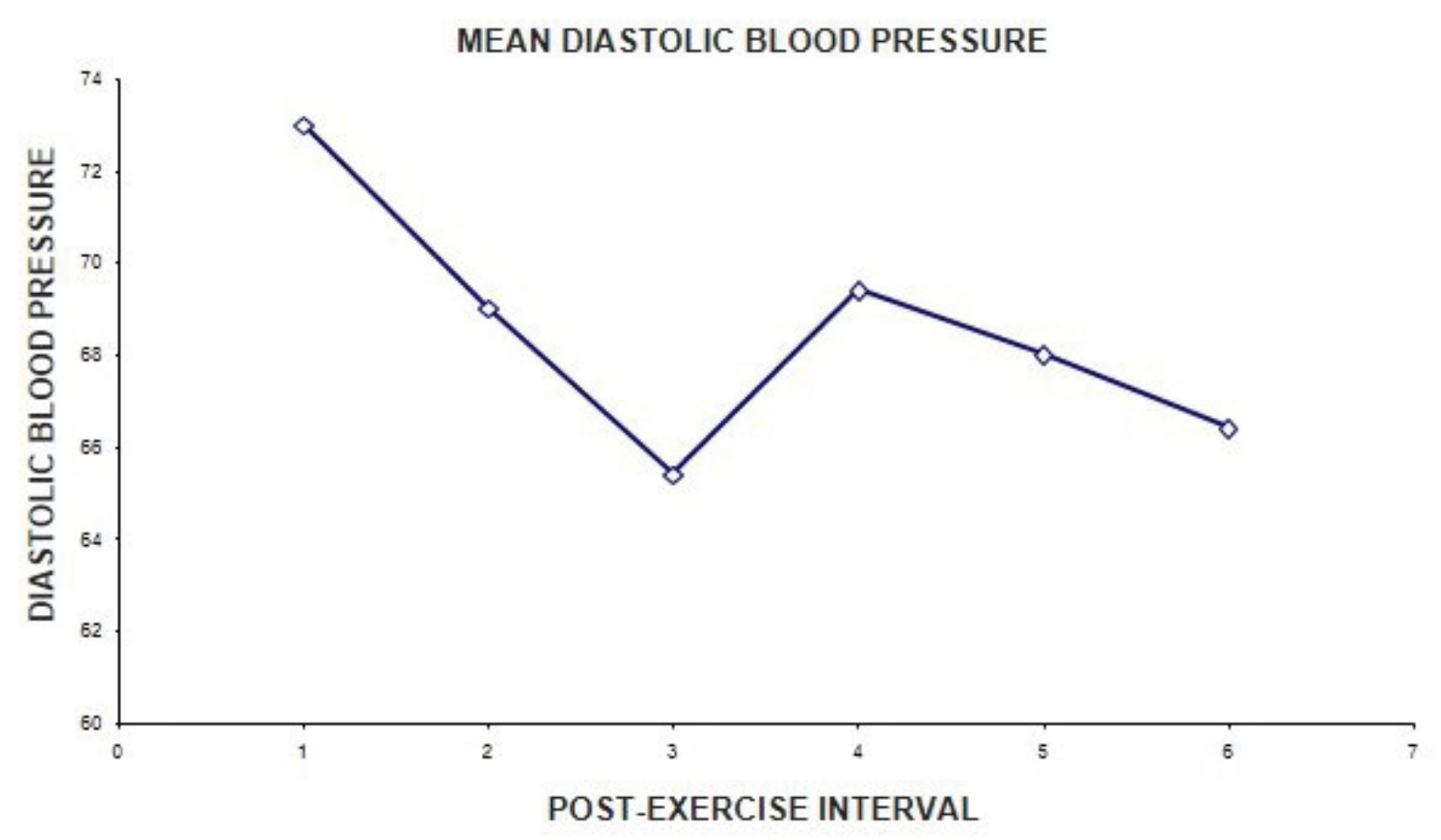

Figure 2: Mean change in diastolic blood pressure of individuals durant and a session concurrent training.

The measurement of blood pressure levels after a single resistance exercise session demonstrates the occurrence of post-exercise hypotension in normal and hypertensive individuals, however, there are controversies regarding the intensity of effort necessary to induce this effect [10]. Regarding aerobic exercise, some studies indicate that the intensity of the activity does not influence the magnitude of the hypotensive effect [4].
There is no consensus in the literature on the effects of the size of the muscle group involved in exercise and its influence on acute cardiovascular responses to resistance exercise [11]. The SBP values in subsequent times the exercise seem to decline quickly, the baroreflex mechanism by hyperemia due to muscle contraction and suppression of sympathetic activity [12].

The results obtained in the study indicate HPE for up 
to 60 minutes when the concurrent training in question is carried out and suggests a reduction in BP similar to aerobic training, however, with a slightly greater reduction in DBP.

The present study is relevant due to the scarcity of information on the subject and its importance in the treatment of arterial hypertension. Emphasizing that concurrent training is common, especially among the elderly and participants in functional and cardiac rehabilitation programs.

\section{Conclusion}

It can be concluded that concurrent aerobic and counter-resistance training at moderate intensity causes HPE for up to 60 minutes, however, there were more marked reductions in SBP levels when compared to DBP levels.

\section{References}

1. Gomes RV, Aoki MS (2005) Creatine supplementation nullifies the adverse effect of endurance exercise on subsequent strength performance. Rev Bras Med Esporte 11: 131-134.

2. Chtara M, Chamari K, Chaouachi M, Chaouachi A, Koubaa D, et al. (2005) Effects of Intra-Session Concurrent Endurance and Strength Training Sequence on Aerobic Performance and Capacity. Br Sports Med 39: 555-560.

3. Ciolac EG, Guimaraes GV (2004) Physical exercise and metabolic syndrome. Rev Bras Med Esporte 10: 4.

4. Polito MD, Farinatti PTV (2006) Blood pressure behavior after counter-resistance exercises: A systematic review of determining variables and possible mechanisms. Rev Bras Med Esporte 12: 6.

5. Monteiro MF, Filho DCS (2004) Physical exercise and blood pressure control. Rev Bras Med Esporte 10: 6.

6. Cunha GA, Rios ACS, Moreno JRB, Campbell CSG, Simoes HG, et al. (2006) Post-exercise hypotension in hypertensive patients submitted to aerobic exercise of varying intensities and constant intensity exercise. Rev Bras Med Esporte 12: 6.

7. Bermudes AMLM, Vassallo DV, Vasquez EC, Lima EG (2003) Ambulatory blood pressure monitoring in normotensive individuals submitted to two unique exercise sessions: Resistance and Aerobic. Arq Bras Cardiol 81: 5764.

8. Fleck SJ, Kraemer WJ (2006) Fundamentals of muscle strength training. Translation Jerri L. Ribeiro. 3ed. - Porto Alegre: Artmed.

9. Polito MD, Farinatti PTV (2003) Considerations on blood pressure measurement in counter-resistance exercises. Rev Bras Med Esporte 9: 1.

10. Umpierre D, Stein R (2007) Hemodynamic and vascular effects of resistance training: implications for cardiovascular disease. Arq Bras Cardiol 89: 256-262.

11. D'assuncao W, Daltro $M$, Simao $R$, Polito $M$, Monteiro W (2007) Acute cardiovascular responses in strength training conducted in exercises for large and small muscle groups. Rev Bras Med Esporte 13: 2.

12. Mediano MFF, Paravidino V, Simao R, Pontes FL, Polito MD (2005) Subacute blood pressure behavior after strength training in controlled hypertensive patients. Rev Bras Med Esporte 11: 6. 